\title{
Serum inhibin B for differentiating between congenital hypogonadotropic hypogonadism and constitutional delay of growth and puberty: a systematic review and meta-analysis
}

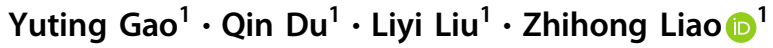 \\ Received: 11 February 2020 / Accepted: 1 December 2020 / Published online: 19 January 2021 \\ (c) The Author(s) 2021
}

\begin{abstract}
Purpose The distinction between congenital hypogonadotropic hypogonadism (CHH) and constitutional delay of growth and puberty (CDGP) in patients with delayed puberty is difficult to distinguish, but important for timely treatment. The aim of this study is to perform a systematic review and meta-analysis to determine the diagnostic performance of serum inhibin B (INHB) levels for differentiating CHH and CDGP.

Methods PubMed, EMBASE, and Cochrane Library databases were systematically searched from the date of database inception to November 10, 2019 for studies examining the use of serum INHB to discriminate between CHH and CDGP. Pooled odds ratios (OR), sensitivity, specificity, and 95\% confidence intervals (CI) were calculated. The Quality Assessment of Diagnostic Studies-2 (QUADAS-2) was used to assess the quality of the included studies. Sub-analyses were performed including that based on testicular volume (TV) and study design.

Results Seven studies, comprising of 349 patients (96 CHH and 253 CDGP), were included in the meta-analysis. For differentiating between $\mathrm{CHH}$ and CDGP, INHB level exhibited good diagnostic accuracy with a pooled sensitivity of 92\% (95\% confidence interval [CI]: $0.86-0.96, I^{2}=0.4 \%, p=0.4343$ ), specificity of $92 \%$ (95\% CI: $0.88-0.94, I^{2}=$ $68.1 \%, p=0.0009$ ), and pooled area under the receiver operating characteristic curve (AUC) of 0.9619 . The cut-off values of INHB for boys were 56, 66, 80, 96, 94.7, 111, and $113 \mathrm{pg} / \mathrm{ml}$ (assay method standardized to Gen II ELISA). Sub-analyses showed that testicular volume and study design could be a source of statistically significant heterogeneity in specificity. In boys with a testicular volume of $\leq 3 \mathrm{ml}$, INHB performed well with a sensitivity of $92 \%$, specificity of 98\%, and AUC of 0.9956.

Conclusion INHB exhibits excellent diagnostic efficiency in distinguishing CHH from CDGP, especially in boys with severe puberty deficiency $(\mathrm{TV} \leq 3 \mathrm{ml})$.
\end{abstract}

Keywords Inhibin B · Congenital hypogonadotropic hypogonadism • Constitutional delay of growth and puberty • Meta-analysis

\section{Introduction}

Delayed puberty (DP) is defined as pubertal onset occurring at an age of 2 or 2.5 standard deviations later than the mean of the population. Classically, it refers to the absence of testicular enlargement (volume $<4 \mathrm{ml}$ ) in boys by age 14

Zhihong Liao

liaozhh@mail.sysu.edu.cn

1 Department of Endocrinology and Metabolism, Hospital of Sun Yat-sen University, Guangzhou, Guangdong, PR China and absent breast development in girls by age 13 [1]. The causes of delayed puberty can be classified into five categories: constitutional delay of growth and puberty (CDGP) (53\%), functional hypogonadotrophic hypogonadism (19\%), hypogonadotrophic hypogonadism (HH) (12\%), hypergonadotrophic hypogonadism (13\%), and unclassified (3\%) [2]. CDGP is the most common reason for delayed puberty, and is a physiological variant of normal puberty characterized by a slowing of growth and delayed timing of pubertal development [3]. Congenital hypogonadotropic hypogonadism $(\mathrm{CHH})$ is a form of $\mathrm{HH}$ that occurs in $\sim 1$ in 4000 births. It is a relatively rare heterogeneous disorder caused by deficient production, secretion, or action 
of gonadotropin-releasing hormone (GnRH). Idiopathic hypogonadotropic hypogonadism (IHH), isolated hypogonadotropic hypogonadism, and isolated GnRH deficiency are similar to $\mathrm{CHH}$, and the terms are often are used interchangeably [4]. The term $\mathrm{CHH}$ is commonly used because of the disorders hereditary characteristics. $\mathrm{CHH}$ and CDGP share several similar signs and symptoms. Differentiating between the two during early adolescence is challenging, especially in normosmic pre-pubertal boys presenting with pubertal delay without cryptorchidism, because patients with partial $\mathrm{CHH}$ present with different degrees of pubertal underdevelopment.

The presence of progressive pubertal development by age 18 is the "gold standard" for differentiating CDGP from $\mathrm{CHH}$. However, an early diagnosis of $\mathrm{CHH}$ or CDGP is important because a delayed diagnosis may be harmful to psychological well-being and quality of life, and because of the impact on bone mineralization and fertility [5]. A variety of methods for differentiating the two disorders have been proposed, including nocturnal luteinizing hormone (LH) sampling, testosterone response to human chorionic gonadotropin (HCG), a GnRH-stimulated LH response, measurement of urinary gonadotrophins, and daily urine excretion of follicle-stimulating hormone (FSH) and LH [6-10]. Most of these tests are invasive, timeconsuming, imprecise, and/or costly. Likewise, it is hard to standardize the various $\mathrm{GnRH}, \mathrm{GnRH}$ agonists (GnRHa), and HCG stimulation tests worldwide. The medication doses, injection frequency, and timing of blood sampling also vary in the stimulation tests [11-13], which also makes the results difficult to compare. No single test has emerged that is reliable, easy to perform, and has acceptable sensitivity and specificity for distinguishing between $\mathrm{CHH}$ and CDGP.

Serum inhibin B (INHB) level is used for distinguishing CHH from CDGP. Using INHB level avoids the need for repeated injections, and its assay method is relatively simple. INHB is produced in the Sertoli cells of the testis in males, and in the granulosa cells of the ovary in females. It belongs to the transforming growth factor- $\beta$ (TGF- $\beta$ ) super family, and regulates the synthesis and secretion of FSH in a negative feedback loop [14]. INHB level reflects Sertoli cell number and function $[15,16]$. In males, INHB peaks shortly after birth, decreases during childhood, and then increases at puberty due to FSH stimulation. It is a better marker of fertility status than FSH and LH [17]. In females, INHB level is related to the number of antral follicles, and reflects the ovarian response to gonadotrophins $[15,16]$. INHB may be used as a regular screening test for certain ovarian cancers (mucinous carcinomas, granulosa cell tumors), especially in post-menopausal women [18].

A number of studies have examined the diagnostic value of serum INHB level for differentiating between $\mathrm{CHH}$ and
CDGP. However, the sensitivity, specificity, and cut-off values reported in studies are inconsistent. Thus, the purpose of this study was to perform a meta-analysis to determine the diagnostic performance of serum INHB level for differentiating $\mathrm{CHH}$ and CDGP.

\section{Methods and materials}

The meta-analysis complied with the Preferred Reporting Items for Systematic Reviews and Meta-Analyses (PRISMA) statement [19].

\section{Data sources and search strategy}

PubMed, EMBASE, and Cochrane Controlled Register databases were searched from inception to November 10 , 2019 for studies examining the use of INHB level for differentiating $\mathrm{CHH}$ and CDGP. Mesh terms and free-text words were matched and used together for database searches using combinations of keywords as follows: "constitutional delay of growth and puberty [MESH] OR constitutional delay OR constitutional growth delay OR delayed puberty OR CDGP OR CDG OR CDP" AND "congenital hypogonadotropic hypogonadism [MESH] OR hypogonadotropic hypogonadism OR isolated GnRH deficiency OR IHH OR CHH OR HH". The reference lists of all relevant articles were also searched to identify additional potentially relevant studies. Two reviewers (GYT and DQ) performed the database searches, and disagreements were resolved through discussion or by consulting a third researcher (LZH). If full text of a study could not be found, we contacted the author or the development agency.

\section{Inclusion and exclusion criteria}

Studies were included if they meet all of the following criteria: (1) Published in English; (2) The study examined the use of serum INHB level for differentiating between $\mathrm{CHH}$ and CDGP; and (3) Studies reported data as true positive (TP), false positive (FP), true negative (TN), and false negative (FN) rates, or reported overall sample size and sensitivity and specificity values which could be used for statistical analysis.

The exclusion criteria were (1) Animal and in vitro studies; (2) Reviews, meta-analysis, and case reports; (3) Studies that reported unrelated statistical and clinical data; (4) Duplicate articles that were updated versions; (5) The purpose of the study was not related to the objectives of this study; and (6) Studies including patients with functional hypothalamus-pituitary-gonadal (HPG) axis impairment. 


\section{Screening and data extraction}

All included studies were imported into ENDNOTE. Studies were first screened by titles and abstracts, and then the full text was assessed using the inclusion and exclusion criteria. The following data were extracted from studies that met the inclusion criteria: name of the first author, publication year, country of publication, journal name, study design, age of participants, whether testosterone priming treatment was performed, testicular volume (TV), INHB measurement method, $\mathrm{CHH}$ diagnostic standard, CDGP diagnostic standard, cut-off values, number of $\mathrm{CHH}$ patients, number of CDGP patients, and TP, FP, TN, and FN. If data were incomplete for the purposes of the metaanalysis, we contacted the author by mail and asked them to provide the necessary information. Extracted data were recorded in Excel. Screening and data extraction were performed independently by two of the authors (GYT and $\mathrm{DQ}$ ), and disagreements were resolved through discussion or by consulting a third researcher (LZH).

\section{Quality assessment}

The quality of the included studies was assessed using the Quality Assessment of Diagnostic Accuracy Studies-2 (QUADAS-2) tool [20]. The tool has four main components: patient selection, index testing, reference standard, and flow and timing. Each component was evaluated for risk of bias, and the first three components were also evaluated for applicability. The QUADAS-2 also provides relevant questions to help rate studies in the abovementioned domains. Quality assessment was independently done by two researchers (GYT and LLY), and disagreements were resolved through discussion or by consultation with a third researcher (LZH).

\section{Statistical analysis}

Meta-Disc version 1.4 (Ramony Cajal Hospital, Madrid, Spain) [21] was used for data analysis. The pooled sensitivity, specificity, diagnostic odds ratio (DOR), and summary receiver operator characteristics (SROC) for INHB in distinguishing the two conditions were calculated. Sensitivity, specificity, and DOR were presented with a $95 \%$ confidence interval (CI), and for the SROC area under the curve the standard error (SE) was calculated. The Q* index, which is the point closest to the ideal top-left corner of the SROC space, is defined as exhibiting the best combination of sensitivity and specificity [22]. The inconsistency index $\left(I^{2}\right)$ was used to evaluate heterogeneity among the studies: an $I^{2}<25 \%$ indicates low heterogeneity, a value of $25-50 \%$ indicates moderate heterogeneity and an $I^{2}>50 \%$ indicates high heterogeneity [23]. When significant heterogeneity was identified $\left(I^{2}>50 \%\right)$ a random-effects model of analysis was used, whereas a fixed-effects model of analysis was used when heterogeneity was low or moderate. The Spearman correlation coefficient was calculated to determine if a threshold effect existed. Meta-regression analysis was then performed to explore the possible sources of heterogeneity in the studies. Statistical significance was defined as a two-tailed value of $p<0.05$.

\section{Results}

\section{Literature search}

A flow diagram of the literature search and study selection process is shown in Fig. 1. The initial database searches yielded 648 records. Of these, 238 were excluded after duplicates were removed, and 370 were excluded after screening the titles and abstracts. Thus, the full texts of 40 articles were reviewed. One article [24] was retrieved from the reference of included review. Finally, seven studies with 349 cases (96 $\mathrm{HH}$ and $253 \mathrm{CDGP}$ ) were included in the meta-analysis. The main reasons for exclusions were duplication of studies between PubMed and the EMBASE Databases, non-diagnostic research, and studies of INHB not relevant to the purposes of this study (Fig. 1).

\section{Study characteristics}

Details of the included studies are summarized in Table 1 . All were diagnostic studies published from 2010 to 2017, and provided sufficient data for the calculations required for this meta-analysis.

\section{Quality assessment}

The quality assessment of the included studies is summarized in Fig. 2. All studies fulfilled the criteria of the index test, i.e., the evaluation of INHB level was blind to the diagnosis. All studies except one used spontaneous complete pubertal development as the reference diagnostic standard. In the study by Binder et al. [25] six boys had a TV between 5 and $8 \mathrm{ml}$ at 24 months of follow-up, which did not fulfill the definition of either CHH or CDGP defined in their study and were therefore considered inappropriate exclusions. In terms of patient selection, all seven studies had a low risk of bias, and mentioned whether they included consecutive patients. However, three of the studies excluded patients for unclear or unreasonable standards [24-26]. With respect to flow and timing bias, other than the abovementioned three studies, one study [24] ignored a $\mathrm{CHH}$ patient (16 CHH patients were included in the table, but only 15 were analyzed) for an unclear reason, which 
Fig. 1 Flow diagram of study selection

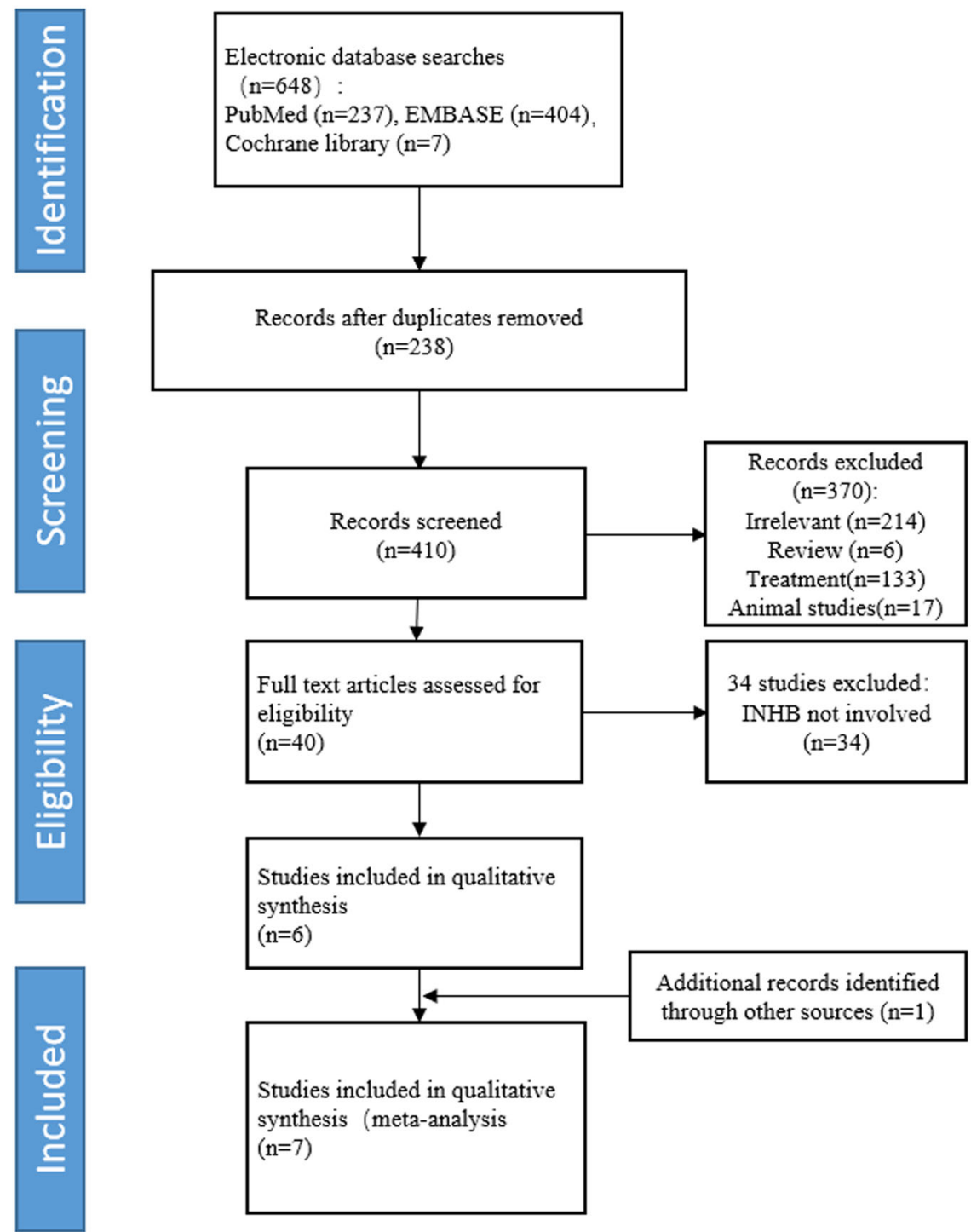

was considered a potential source of bias. Overall, none of the seven studies were excluded from this meta-analysis due to methodological flaws.

\section{Accuracy of INHB for distinguishing CHH and CDGP}

The diagnostic performance results for serum INHB level in differentiating $\mathrm{CHH}$ from CDGP are shown in Fig. 3. The pooled sensitivity and specificity (random-effect model) for serum INHB in distinguishing the two conditions were 0.92 (95\% CI: 0.86-0.96) and 0.92 (95\% CI: 0.88-0.94), respectively. There was a considerable level of heterogeneity for the sensitivity $\left(I^{2}=0.4 \%, p=0.4343\right)$ and for specificity $\left(I^{2}=68.1 \%, p=0.0009\right)$. The pooled DOR was 104.27 (95\% CI: 47.74-227.73), with low heterogeneity $\left(I^{2}=0.00 \%, p=0.6158\right)$. The pooled positive likelihood ratio (PLR) and negative likelihood ratio (NLR) were 9.33 (95\% CI: $5.34-16.31, I^{2}=46.2 \%, p=0.0533$ ) and 0.10
(95\% CI: $0.06-0.18, I^{2}=0.0 \%, p=0.9062$ ), respectively. The AUC was 0.9619, and the $Q^{*}$ was 0.9073. The Spearman correlation coefficient was $-0.417(p=0.3082)$, suggesting no threshold effect existed.

\section{Exploration of heterogeneity}

In the pooled analysis, the included studies were statistically heterogeneous in their estimate of specificity. Potential heterogeneity in sensitivity and specificity was explored with subgroup analysis. TV, study design, methodological quality, publication year, and gender were used as covariants in subgroup analysis. The results suggested that TV and study design could influence the diagnostic value. It is worth noting that subgroup analysis by TV revealed that the pooled sensitivity, specificity, and AUC in $\mathrm{CHH}$ subjects with profound gonadotropin deficiency (as indicated by $\mathrm{TV} \leq 3 \mathrm{ml}$ ) were $0.92,0.98$, and 0.9956 , respectively, which 


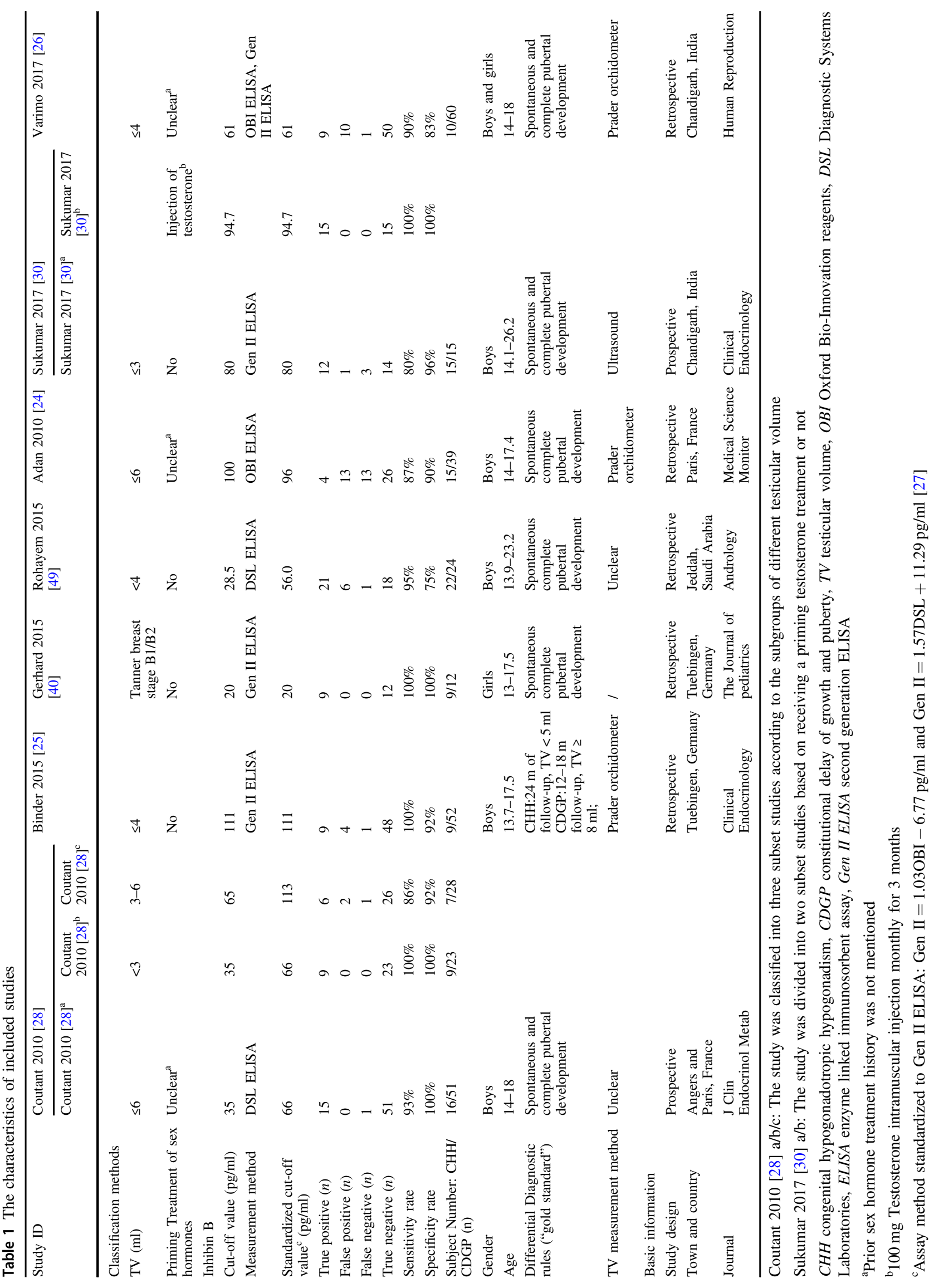




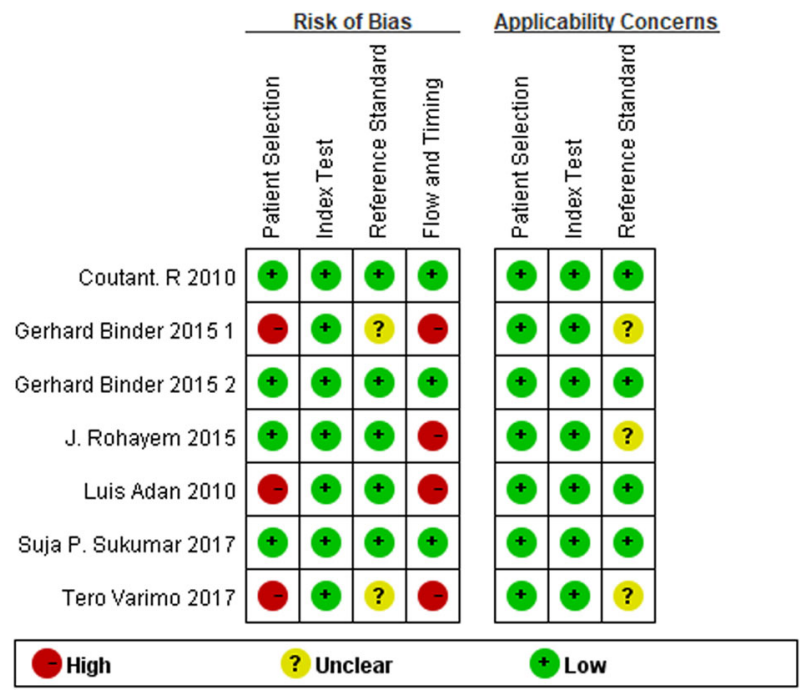

Fig. 2 Quality assessment of the included studies using QUADAS-2 tool. Key: +, low risk; -, high risk; ?, unclear risk

exhibited the best diagnostic efficacy with low heterogeneity of specificity. The pooled sensitivity and specificity of prospective studies were 0.93 and 0.99 , respectively, with lower heterogeneity. The pooled sensitivity, specificity, and AUC for serum INHB in the boys with delayed puberty were $0.92,0.93$, and 0.9645 , respectively. Details of subgroup analyses are shown in Table 2 .

\section{Publication bias}

Since $\mathrm{CHH}$ is a rare disease, the number of studies in each method group was small $(<10)$, and it was difficult to draw funnel plots to examine publication bias. An exhaustive search was performed in the PubMed, EMBASE, and Cochrane Controlled Register databases, and references of related issues were examined to minimize publication bias.

\section{Discussion}

During early adolescence, distinguishing $\mathrm{CHH}$ and CDGP is extremely challenging, as a delay in puberty is a hallmark of both diseases and both have $\mathrm{HH}$ as a characteristic. Whereas GnRH deficiency is permanent in most cases of $\mathrm{CHH}, \mathrm{CDGP}$ is a state of transient GnRH deficiency where puberty eventually begins and is completed without hormonal treatment [1]. In addition, CDGP is a common cause of delayed puberty, whereas $\mathrm{CHH}$ is considerably rarer. Differentiating CHH from CDGP is crucial to avoid postponing hormonal therapy and alleviate the psychological burden associated with delayed sexual maturation [5]. In addition, differentiating a transient condition from a chronic disease can influence a patient's quality of life [5].
This meta-analysis indicated that serum INHB level has good diagnostic accuracy for differentiating the two conditions, with a pooled sensitivity of $92 \%$, specificity of $92 \%$, and a pooled AUC of 0.9619 . It also performed especially well in boys with severe puberty deficiency (TV $\leq 3 \mathrm{ml}$ ) with a sensitivity of $92 \%$, specificity of $98 \%$, and AUC of 0.9956 . The INHB DOR was 104.27, suggesting it is a useful method to identify CHH. A PLR of 9.33 determined in this meta-analysis indicated that patients with an INHB level lower than the cut-off value were nine times more likely to have CHH. In contrast, an NLR of 0.10 suggested that an IHNB level higher than the cut-off value was associated with a $90 \%$ chance of having CDGP.

\section{Variation in the cut-off value of INHB}

In this study, we examined seven cut-off values of INHB level in boys with DP $(28.5 \mathrm{pg} / \mathrm{ml}, 35 \mathrm{pg} / \mathrm{ml}, 65 \mathrm{pg} / \mathrm{ml}$, $80 \mathrm{pg} / \mathrm{ml}, 94.7 \mathrm{pg} / \mathrm{ml}, 100 \mathrm{pg} / \mathrm{ml}$, and $111 \mathrm{pg} / \mathrm{ml})$. Each of the cut-off values exhibited good diagnostic accuracy. The best diagnostic accuracies were observed in the case of severe puberty deficiency (TV $\leq 3 \mathrm{ml})$ with the cut-off value of $35 \mathrm{pg} / \mathrm{ml}$, and in the situation of discontinuing " 3 -month testosterone priming" with a cut-off value of $94.7 \mathrm{pg} / \mathrm{ml}$; in both instances the sensitivity and specificity were $100 \%$. There are several reasons for choosing the various cut-off values.

The first reason is the variation between INHB measurement methods, instruments, and lab techniques. In order to minimize the variation, the Oxford Bio-Innovation reagents (OBI) assay and Diagnostic Systems Laboratories (DSL) assay were standardized to the second-generation enzyme-linked immunosorbent assay (Gen II ELISA). The conversion formulas used were Gen $\mathrm{II}=1.03 \times \mathrm{OBI}-$ $6.77 \mathrm{pg} / \mathrm{ml} ; \quad$ Gen $\quad \mathrm{II}=1.57 \times \mathrm{DSL}+11.29 \mathrm{pg} / \mathrm{ml} \quad$ [27]. Hence, the converted cut-off values are $56 \mathrm{pg} / \mathrm{ml}, 66 \mathrm{pg} / \mathrm{ml}$, $80 \mathrm{pg} / \mathrm{ml}, 96 \mathrm{pg} / \mathrm{ml}, 94.7 \mathrm{pg} / \mathrm{ml}, 111 \mathrm{pg} / \mathrm{ml}$, and $113 \mathrm{pg} / \mathrm{ml}$, which narrowed the range of INHB for differentiating CHH and CDGP (Table 1).

Secondly, different baseline TV greatly impacted the diagnostic cut-off value of INHB. The results of subgroup analysis suggested that heterogeneity mainly stemmed from the TV value, and also suggested that TV might influence the cut-off value. In the study by Coutant et al. [28], the cutoff value in boys was $35 \mathrm{pg} / \mathrm{ml}$ at genital stage 1 , and $65 \mathrm{pg} /$ $\mathrm{ml}$ at stage 2. INHB is secreted by Sertoli cells and reflects Sertoli cell number and function, which correlates well with testicular size. A TV $\leq 3 \mathrm{ml}$ indicates that the patient has profound gonadotropin deficiency and a relatively lower serum INHB level. Another important reason for using different cut-off values is the TV assessment method. The measurement of TV can vary markedly between different examiners and measurement methods. It is usually 


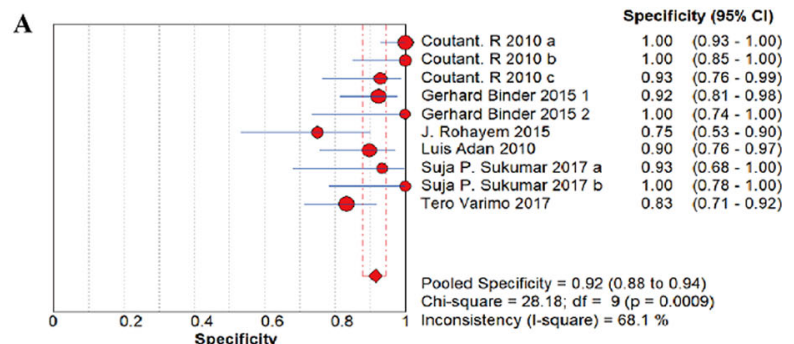

B

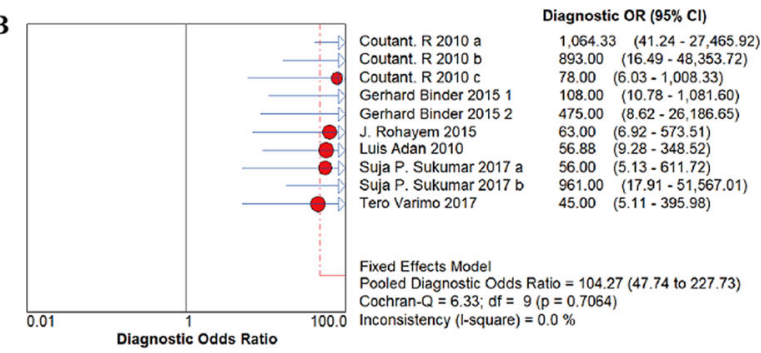

C

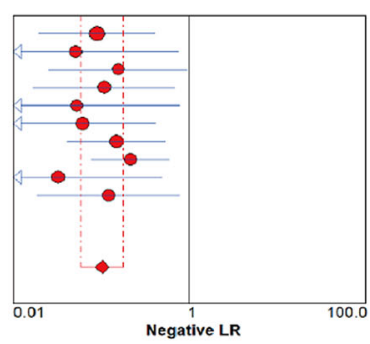

Coutant. R 2010 Coutant. R 2010 b $0.05(0.00-0.76)$

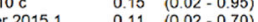
$\begin{array}{lll} & 0.11 & (0.02-0.70)\end{array}$ Rohayem 2015 Lis Adan 2010

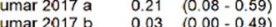
Suja P. Sukumar 2017

Fixed Effects Mode! Doled Negative LR $=0.10(0.06$ to 0.18$)$ Cochran- $Q=4.08 ;$ df $=9(p=0.9062)$
nconsistency $(1$-square $)=0.0 \%$

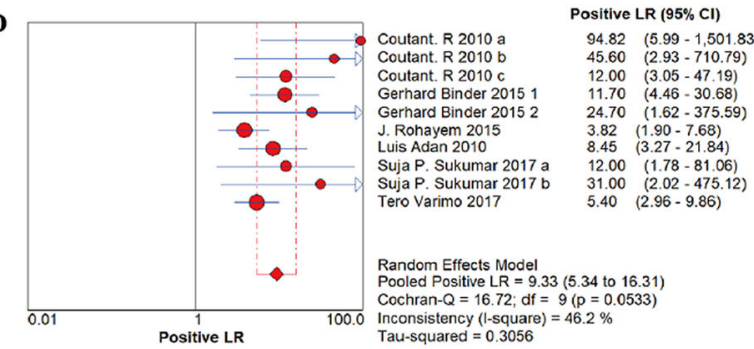

Fig. 3 Forest plot for sensitivity (a), specificity (b), Positive likelihood ratio (LR) (c), Negative LR (d), pooled diagnostic odds ratio (DOR) (e), of eligible studies for INHB in diagnosis. The results are

suggested that there are only a few designated examiners to perform TV assessment with a Prader orchidometer. In our experience, the TV measured with a Prader orchidometer is overestimated by $4.5 \pm 2.7 \mathrm{ml}$ ( 3.2 times) compared to the ultrasonographic calculation of TV in CHH [29].

Thirdly, the cut-off value is affected by different sampling conditions; a naïve assessment without any intervention treatment, or after testosterone treatment. Sukumar et al. [30] demonstrated that an INHB level $>94.7 \mathrm{pg} / \mathrm{ml}$ was discriminatory for diagnosing CDGP after withdrawal of testosterone priming, with a sensitivity of $100 \%$ and specificity of $100 \%$. On the other hand, basal INHB levels $>80 \mathrm{ng} / \mathrm{ml}$ prior to testosterone therapy had a sensitivity of $80 \%$ and specificity of $96 \%$. Thus, prior treatment with sex
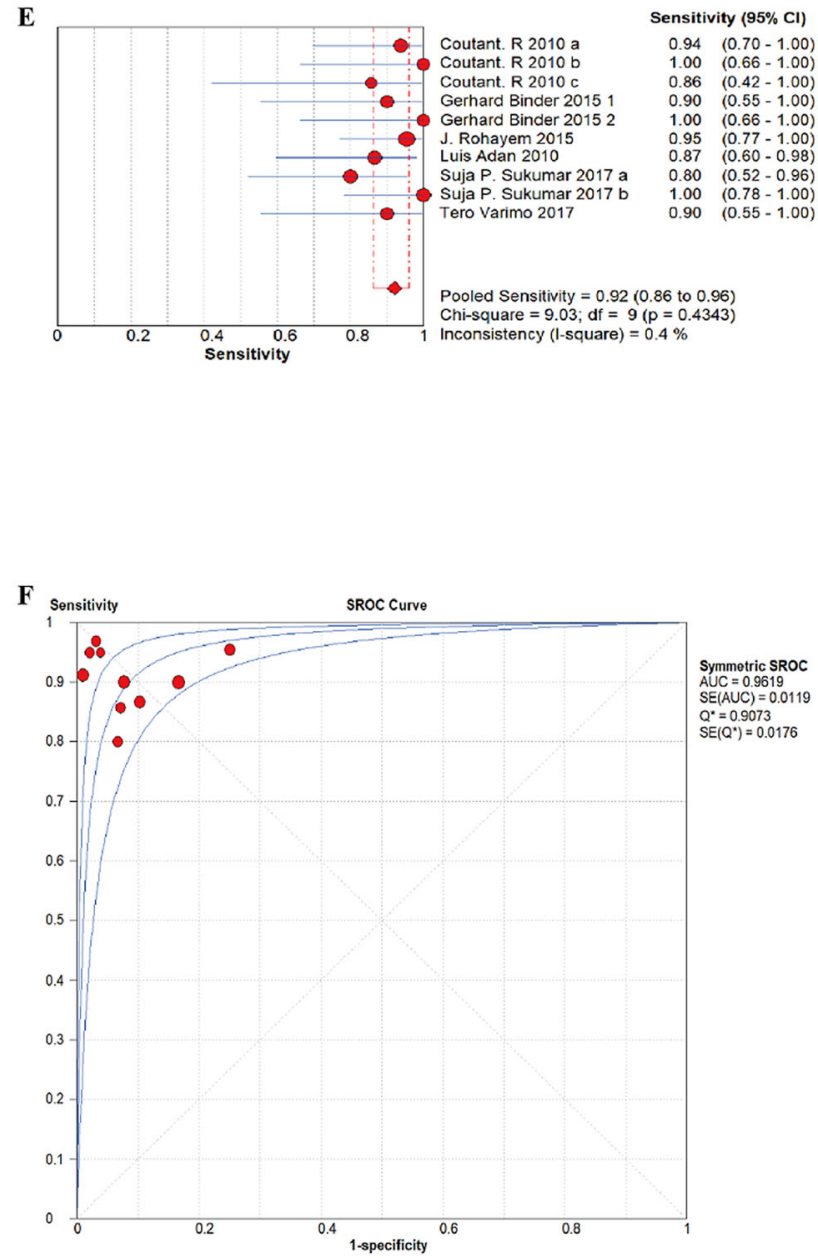

represented with study estimate, summary receiver-operating characteristic (sROC) curves and $95 \%$ confidence region (f)

hormones might influence the cut-off value of INHB to some extent. Prior treatment with sex hormones might vary in these studies.

Lastly, $\mathrm{CHH}$ is a very heterogeneous disorder and includes reversal of $\mathrm{CHH}$ [31, 32], partial $\mathrm{CHH}$ [33], and $\mathrm{CHH}$ occurring in adult patients after normal pubertal development [34], which makes its diagnosis complicated. $\mathrm{CHH}$ patients might have normal adult testosterone levels after discontinuing hormonal therapy, otherwise known as reversal of $\mathrm{CHH}$. Reversibility occurs in both males and females with $\mathrm{CHH}$, and it is more common (10-20\% in males, and a few case reports for females) than previously thought [32]. Partial $\mathrm{CHH}$ typically presents with mild gonadotropin deficiency and partial puberty development [33]. 
Table 2 Summary results of subgroup analysis for INHB in the diagnosis between $\mathrm{CHH}$ and CDGP

\begin{tabular}{|c|c|c|c|c|c|c|c|}
\hline \multirow[t]{2}{*}{ Categorical variable } & \multirow{2}{*}{$\begin{array}{l}\text { No. of } \\
\text { studies }\end{array}$} & \multicolumn{3}{|l|}{ Sensitivity } & \multicolumn{3}{|l|}{ Specificity } \\
\hline & & $95 \% \mathrm{CI}$ & $I^{2}(\%)$ & $P$ value & $95 \% \mathrm{CI}$ & $I^{2}(\%)$ & $P$ value \\
\hline \multicolumn{8}{|l|}{ Testicular volume(ml) } \\
\hline$\leq 3$ & 3 & $0.92(0.79-0.98)$ & 67.4 & 0.0464 & $0.98(0.90-1.00)$ & 22.3 & 0.2761 \\
\hline$\leq 4$ & 3 & $0.93(0.81-0.99)$ & 0.00 & 0.7884 & $0.85(0.78-0.91)$ & 53.7 & 0.1155 \\
\hline$\leq 6$ & 2 & $0.90(0.74-0.98)$ & 0.00 & 0.5021 & $0.96(0.89-0.99)$ & 85.6 & 0.0085 \\
\hline \multicolumn{8}{|l|}{ Study design } \\
\hline Prospective & 4 & $0.93(0.82-0.98)$ & 51.4 & 0.1033 & $0.99(0.95-1.00)$ & 23.7 & 0.2690 \\
\hline Retrospective & 5 & $0.92(0.83-0.97)$ & 0.00 & 0.6457 & $0.87(0.82-0.92)$ & 51.6 & 0.0822 \\
\hline \multicolumn{8}{|l|}{ Methodological quality } \\
\hline Low risk & 5 & $0.94(0.85-0.98)$ & 46.2 & 0.1147 & $0.99(0.95-1.00)$ & 3.6 & 0.3860 \\
\hline Not low risk & 4 & $0.91(0.81-0.97)$ & 0.00 & 0.8098 & $0.86(0.80-0.91)$ & 38.2 & 0.1828 \\
\hline \multicolumn{8}{|l|}{ Publication year } \\
\hline 2010 & 3 & $0.93(0.80-0.98)$ & 2.40 & 0.3589 & $0.96(0.91-0.99)$ & 77.3 & 0.0123 \\
\hline 2015 & 3 & $0.95(0.83-0.99)$ & 0.00 & 0.5104 & $0.89(0.80-0.94)$ & 71.9 & 0.0285 \\
\hline 2017 & 3 & $0.90(0.76-0.97)$ & 55.5 & 0.1058 & $0.88(0.79-0.94)$ & 63.1 & 0.0664 \\
\hline \multicolumn{8}{|l|}{ Gender } \\
\hline Boys only & 7 & $0.92(0.85-0.97)$ & 16.4 & 0.3052 & $0.93(0.89-0.96)$ & 71.5 & 0.0018 \\
\hline Girls included & 2 & $0.95(0.74-1.00)$ & 25.0 & 0.2482 & $0.86(0.76-0.93)$ & 74.7 & 0.0467 \\
\hline
\end{tabular}

The cut-off value for INHB in boys ranged from 56 to $113 \mathrm{pg} / \mathrm{ml}$ (Gen II ELISA), suggesting a variance resulting from the patient's condition and hospital laboratory testing variances. Although this study could not confirm a single, optimal cut-off value, the results do indicate that IHNB is an excellent diagnostic marker in differentiating $\mathrm{CHH}$ from CDGP, and that every center should determine its own cutoff values of INHB.

\section{INHB in girls with CHH or CDGP}

In contrast to boys with DP, there is a paucity of studies of girls with DP [35]. Most studies included in this metaanalysis were focused on prepubertal boys. The prevalence of girls with CDGP is approximately five times lower than that of boys [36]. Only one randomized study included several girls with $\mathrm{CHH}$ [37]. Several articles have reported a lower INHB concentration in prepubertal girls [38, 39]. Binder et al. [40] demonstrated a basal INHB cut-off of $20 \mathrm{pg} / \mathrm{ml}$ (Gen II ELISA). Similar results were reported by Juul et al. [41], which reported a lower reference range of $20 \mathrm{pg} / \mathrm{ml}$ for INHB for healthy girls at Tanner stage B2.

\section{Recent studies examining the discrimination of $\mathrm{CHH}$ from CDGP}

We reviewed some features that may assist in the differential diagnosis, noting that although individual indicators may not provide a definitive resolution, a combination of multiple indicators and clinical observations can strengthen arguments for or against a particular diagnosis. New investigations regarding parameters for distinguishing $\mathrm{CHH}$ and CDGP are summarized below.

Some clinical features can potentially distinguish CDGP from $\mathrm{CHH}$, although they do not have high diagnostic value. The presence of cryptorchidism and/or micropenis favors a diagnosis of $\mathrm{CHH}$, and indicates the absence of gonadotropins and sexual hormones during both fetal life and minipuberty $[1,30]$. Congenital hyposmia or anosmia are not consistent with a diagnosis of CDGP [42]. TV is useful for discriminating $\mathrm{CHH}$ from CDGP in boys. In a study of 174 boys with DP [30], those with a TV $<1.1 \mathrm{ml}$ (measured by Prader orchidometer) showed a 100\% sensitivity and $91 \%$ specificity in distinguishing $\mathrm{CHH}$ from CDGP. Growth velocity has been shown to offer no additional diagnostic value in distinguishing between CDGP and $\mathrm{CHH}$ [43]. Family history is also not helpful for distinguishing the two conditions because individuals with $\mathrm{CHH}$ often have family members with CDGP [44].

Genetic testing shows promise for distinguishing the two conditions, though more studies are needed. Cassatella et al. [45] suggested that CDGP and $\mathrm{CHH}$ have distinct genetic profiles; $\mathrm{CHH}$ has gene mutations in $51 \%$ of the probands, but CDGP has mutations in only $7 \%$ of probands.

It is challenging to make a clinical distinction between $\mathrm{CHH}$ and CDGP based on other biochemical markers because most biochemical markers are not always discriminatory. To date, there is no biochemical marker that can fully differentiate $\mathrm{CHH}$ from CDGP in early adolescences. Harrington and Palmert [46] systematically 
reviewed studies of basal gonadotropin levels (e.g., LH, $\mathrm{FSH}$, testosterone), and reported that gonadotropin levels had limited ability in distinguishing between $\mathrm{CHH}$ and CDGP, primarily because of the overlap of gonadotropin levels. There had been many attempts to use stimulation tests, including GnRH and GnRHa stimulation tests, and HCG testing, to distinguish between the two conditions. A $\mathrm{GnRH}$ test has been considered useful for identifying $\mathrm{CHH}$; $\mathrm{CHH}$ is highly probable when a GnRH-stimulated $\mathrm{LH}$ response is blunted. Varimo et al. [26] demonstrated a peak LH cut-off value of $4.3 \mathrm{IU} / \mathrm{L}$ post $\mathrm{GnRH}$ stimulation test had a sensitivity of $100 \%$ and specificity of $75 \%$ for detecting CHH. Another study [47] showed that a peak LH $<9.74$ IU/L had moderate sensitivity $(80.0 \%)$ and specificity $(86.4 \%)$ for diagnosing $\mathrm{CHH}$ in males. However, different stimulation methods with GnRH or GnRHa, and different dosages of GnRHa have resulted in a variety of peak LH cut-off values [9, 48]. In HCG stimulation tests, different HCG dosages, injection frequency, and timing of blood sampling affect the results of testosterone measurements [11-13]. In a study of $14 \mathrm{CHH}$ and 29 CDGP patients [46], HCG 1500 IU was prescribed on days 1, 3, and 4 and testosterone level was measured on days 1 and 5. A testosterone level of $<1.04 \mu \mathrm{g} / \mathrm{L}$ on day 5 showed a $92 \%$ sensitivity and $92 \%$ specificity for diagnosis. When HCG 1500 IU was injected on days $1,3,4,9,12,16$, and 19 and testosterone was measured on days 1,5 , and 20 a testosterone level of $<2.75 \mu \mathrm{g} / \mathrm{L}$ on day 20 exhibited a $92 \%$ sensitivity and $95 \%$ specificity for distinguishing $\mathrm{CHH}$ from CDGP. All of the aforementioned results are inferior to that of using a baseline INHB level, which was found to have $93 \%$ sensitivity and $100 \%$ specificity in this meta-analysis.

The frequent injections and samplings of stimulation tests make them impractical for routine use. Some studies have examined the diagnostic utility of anti-Mùllerian hormone (AMH), which is secreted by Sertoli cells. A lower $\mathrm{AMH}$ level is suggestive of $\mathrm{CHH}$, but it is less accurate than INHB. However, AMH had distinctive efficacy for prepubertal boys with a bilateral $\mathrm{TV} \leq 8 \mathrm{ml}$. AMH levels are significantly higher in CDGP boys than $\mathrm{CHH}$ boys during pre-puberty [49]. Normally, AMH levels do not change in a linear manner over the pubertal transition period, but rather fluctuate $[28,30]$. AMH declines transiently after birth, then increases to a peak at 2-4 years old and remains elevated during pre-puberty. Afterward, it decreases sharply with puberty development. There was an overlap of AMH levels between subgroups, which is likely because of the lower diagnostic efficacy of AMH values relative to INHB in distinguishing CDGP and $\mathrm{CHH}$. Coutant [28] described a cut-off value of $110 \mathrm{pmol} / \mathrm{l}(15.4 \mathrm{ng} / \mathrm{ml})$ analyzed by electrochemiluminescence immunoassay (ECLIA). Rohayem et al. [49] showed a cut-off value of $20 \mathrm{ng} / \mathrm{ml}$ using an ELISA could differentiate between CDGP and $\mathrm{CHH}$. Other markers such as insulin-like factor (INSL), dehydroepiandrosterone sulfate, and insulin-like growth factor (IGF)-1 did not improve the accuracy of diagnosis [50].

Coutant et al. [28] found that the combination of INHB and LH (with cut-offs of $35 \mathrm{pg} / \mathrm{ml}$ and $0.5 \mathrm{mIU} / \mathrm{L}$, respectively) offered $100 \%$ sensitivity and specificity in differentiating between $\mathrm{CHH}$ and CDGP in adolescents with a $\mathrm{TV} \leq 3 \mathrm{ml}$. According to our meta-analysis results, and a comparison of the results with the above-mentioned approaches, a single measurement of INHB level provides good diagnostic accuracy and is far less costly.

\section{Strengths and limitations}

This was the first meta-analysis that comprehensively explored the diagnostic efficiency of INHB in distinguishing $\mathrm{CHH}$ from CDGP, and provided a quantitative analysis. However, this study had some limitations. The number of studies and patients was relatively low because $\mathrm{CHH}$ is a rare disease, and some studies had low methodological quality. We could not determine a single, optimal cut-off value. Less than ten studies were included in the meta-analysis, and thus the results of the subgroup analyses must be treated with caution. Lastly, potential publication bias might exist because only studies published in English were included.

\section{Conclusion}

INHB level is useful for differentiation between $\mathrm{CHH}$ and CDGP, especially in boys with PD and a TV $\leq 3 \mathrm{ml}$. An INHB reference range of $56-113 \mathrm{pg} / \mathrm{ml}$ for boys and $20 \mathrm{pg} /$ $\mathrm{ml}$ for girls (Gen II ELISA) may provide useful information for discrimination between $\mathrm{CHH}$ and CDGP. Additional studies with large sample sizes and standardized methodology would be required to achieve a more robust and credible result. A combination of multiple parameters, such as clinical characteristics, laboratory data, and genetic sequencing may ultimately provide high diagnostic accuracy and determination of prognosis.

Funding Guangzhou Science and Technology Programme, Grant/ Award Number: 201604020090.

\section{Compliance with ethical standards}

Conflict of interest The authors declare that they have no conflict of interest.

Ethical approval As this meta-analysis was performed based on the published data, ethical approval was not required.

Publisher's note Springer Nature remains neutral with regard to jurisdictional claims in published maps and institutional affiliations. 
Open Access This article is licensed under a Creative Commons Attribution 4.0 International License, which permits use, sharing, adaptation, distribution and reproduction in any medium or format, as long as you give appropriate credit to the original author(s) and the source, provide a link to the Creative Commons license, and indicate if changes were made. The images or other third party material in this article are included in the article's Creative Commons license, unless indicated otherwise in a credit line to the material. If material is not included in the article's Creative Commons license and your intended use is not permitted by statutory regulation or exceeds the permitted use, you will need to obtain permission directly from the copyright holder. To view a copy of this license, visit http://creativecommons. org/licenses/by/4.0/.

\section{References}

1. M.R. Palmert, L. Dunkel, Delayed puberty. N. Engl. J. Med. 366 (5), 443-453 (2012)

2. C. Wei, E.C. Crowne, Recent advances in the understanding and management of delayed puberty. Arch. Dis. Childhood. (2016) https://doi.org/10.1136/archdischild-2014-307963

3. A. Soliman, V.D. Sanctis, An approach to constitutional delay of growth and puberty. Indian J. Endocrinol. Metab. 16(5), 698 (2012)

4. D. Cassatella, S.R. Howard, J.S. Acierno et al. Congenital hypogonadotropic hypogonadism and constitutional delay of growth and puberty have distinct genetic architectures. Eur. J. Endocrinol. 178(4), 377-388 (2018)

5. U. Boehm, P.M. Bouloux, M.T. Dattani et al. Expert consensus document: European Consensus Statement on congenital hypogonadotropic hypogonadism-pathogenesis, diagnosis and treatment. Nat. Rev. Endocrinol. 11(9), 547-564 (2015).

6. M. Grumbach, DM Styne. Puberty: ontogeny, neuroendocrinology, physiology, and disorders. in: Williams Textbook of Endocrinology, 10th edn. ed. by P.R. Larsen, H.M. Kronenberg, S. Melmed, K.S. Polonsky, (Philadelphia: Saunders, 2003), pp. $1115-1286$

7. D.C. Brown, H.F. Stirling, G.E. Butler et al. Differentiation of normal male prepuberty and hypogonadotrophic hypogonadism using an ultrasensitive luteinizing hormone assay. Horm. Res. Paediatrics 46(2), 83-87 (1996)

8. I.M. Spitz, H.J. Hirsch, S. Trestian, The prolactin response to thyrotropin-releasing hormone differentiates isolated gonadotropin deficiency from delayed puberty. N. Engl. J. Med. 308(10), 575-579 (1983)

9. L. Dunkel, GnRH and HCG tests are both necessary in differential diagnosis of male delayed puberty. Arch. Pediatrics Adolesc. Med. 139(5), 494 (1985)

10. H. Kulin, L. Demers, V. Chinchilli et al. Usefulness of sequential urinary follicle-stimulating hormone and luteinizing hormone measurements in the diagnosis of adolescent hypogonadotropism in males. J. Clin. Endocrinol. Metab. 78(5), 1208-1211 (1994)

11. L. Dunkel, J. Perheentupa, R. Sorva, Single versus repeated dose human chorionic gonadotropin stimulation in the differential diagnosis of hypogonadotropic hypogonadism. J. Clin. Endocrinol. Metab. 60(2), 333-337 (1985)

12. T.F. Kolon, O.F. Miller, Comparison of single versus multiple dose regimens for the human chorionic gonadotropin stimulatory test. J. Urol. 166(4), 1451-1454 (2001)

13. J. Dixon, A.M. Wallace, S. O“Toole et al. Prolonged human chorionic gonadotrophin stimulation as a tool for investigating and managing undescended testes. Clin. Endocrinol. 67(6), 816-821 (2007)
14. C.Y. Andersen, K.T. Schmidt, S.G. Kristensen, M. Rosendahl, A. G. Byskov, E. Ernst, Concentrations of AMH and inhibin-B in relation to follicular diameter in normal human small antral follicles. Hum. Reprod. 25(5), 1282-1287 (2010)

15. N. Lahlou, M. Roger, Inhibin B in pubertal development and pubertal disorders. Semin Reprod. Med. 22, 165-175 (2004)

16. S.J. Meachem, E. Nieschlag, M. Simoni. Inhibin B in male reproduction: pathophysiology and clinical relevance. Eur. J. Endocrinol. 145, 561-571 (2001)

17. P. Kumanov, K. Nandipati, A. Tomova, A. Agarwal, Inhibin B is a better marker of spermatogenesis than other hormones in the evaluation of male factor infertility. FertilSteril 86(2), 332-338 (2006)

18. C.H. Mom, M.J. Engelen, P.H. Willemse et al. Granulosa cell tumors of the ovary: the clinical value of serum inhibin A and B levels in a large single center cohort. Gynecol. Oncol. 105(2), 365-372 (2007)

19. Karsten. Preferred reporting items for systematic reviews and meta-analyses (PRISMA) statement and publication bias. J. Cranio Maxillofacial Surgery. 39(2), 91-92 (2011)

20. P.F. Whiting, A.W.S. Rutjes, M.E. Westwood, S. Mallett, J.J. Deeks, J.B. Reitsma, M.M.G. Leeflang, J.A.C. Sterne, P.M.M. Bossuyt, QUADAS-2 Group. QUADAS-2: a revised tool for the quality assessment of diagnostic accuracy studies. Ann. Intern. Med. 155, 529-536 (2011)

21. J. Zamora, A. Muriel, K. Khan, A Meta-DiSc: a software for meta-analysis of test accuracy data. BMC Med. Res. Methodol. 6, $31(2006)$

22. S.D. Walter, Properties of the summary receiver operating characteristic (SROC) curve for diagnostic test data. Stat. Med 21(9), 1237-1256 (2002)

23. J.P. Higgins, S. Green. Cochrane Handbook for Systematic Reviews of Interventions. Version 5.1.0. (The Cochrane Collaboration, 2011), https://www.handbook.cochrane.org.

24. L. Adan, LechevalierP, A.C. Couto-Silva et al. Plasma inhibin B and antimüllerian hormone concentrations in boys: discriminating between congenital hypogonadotropic hypogonadism and constitutional pubertal delay. Med. Sci. Monit. 16(11), 511-517 (2010)

25. G. Binder, R. Schweizer, G. Blumenstock et al. Inhibin B plus LH vs $\mathrm{GnRH}$ agonist test for distinguishing constitutional delay of growth and puberty from isolated hypogonadotropic hypogonadism in boys. Clin. Endocrinol. (Oxf.) 82(1), 100-105 (2015)

26. T. Varimo, J.M. Päivi, K. Johanna et al. Congenital hypogonadotropic hypogonadism, functional hypogonadotropism or constitutional delay of growth and puberty? An analysis of a large patient series from a single tertiary center. Hum. Reprod. 32(1), 147 (2017)

27. B. Kalra et al. "Development of a second generation Inhibin B ELISA.”. J. Immun. Methods 362(1-2), 22-31 (2010)

28. B.E. RégisCoutant, C. Bouvattier et al. Baseline inhibin B and anti-Mullerian hormone measurements for diagnosis of hypogonadotropic hypogonadism $(\mathrm{HH})$ in boys with delayed puberty. J. Clin. Endocrinol. Metab. 95(12), 5225-5232 (2010)

29. W.A.N.G. Yu, W.A.N.G. Wei, J.I. Wen et al. In patients with hypogonadotropin-induced hypogonadism, the prader testis has a greater error. Med. Inf. 11, 33-35 (2017)

30. S.P. Sukumar, A. Bhansali, N. Sachdeva et al. Diagnostic utility of testosterone priming prior to dynamic tests to differentiate constitutional delay in puberty from isolated hypogonadotropic hypogonadism. Clin. Endocrinol. 86(5), 717-724 (2017)

31. V.F. Sidhoum, Y.M. Chan, M.F. Lippincott et al. Reversal and relapse of hypogonadotropic hypogonadism: resilience and fragility of the reproductive neuroendocrine system. J. Clin. Endocrinol. Metab. 99(3), 861-870 (2014)

32. A.A. Dwyer, T. Raivio, N. Pitteloud, Management of endocrine disease: reversible hypogonadotropic hypogonadism. Eur. J. Endocrinol. 174(6), R267-R274 (2016) 
33. D. Beneduzzi, E.B. Trarbach, L. Min, A.A. Jorge, H.M. Garmes et al. Role of gonadotropin-releasing hormone receptor mutations in patients with a wide spectrum of pubertal delay. FertilSteril. 102, 838-846 (2014)

34. L.B. Nachtigall, P.A. Boepple, F.P. Pralong, Crowley JrWF: Adultonset isolated hypogonadotropic hypogonadism - a treatable form of male infertility. N. Engl. J. Med. 336, 410-415 (1997)

35. J. Harrington, M.R. Palmert, Clinical review: distinguishing constitutional delay of growth and puberty from isolated hypogonadotropic hypogonadism: critical appraisal of available diagnostic tests. J. Clin. Endocrinol. Metab. 97, 3056-3067 (2012)

36. I.L. Sedlmeyer, M.R. Palmert, Delayed puberty: analysis of a large case series from an academic center. J. Clin. Endocrinol. Metab. 87, 1613-1620 (2002)

37. N.E. Skakkebaek, J. Bancroft, D.W. Davidson, P. Warner, Androgen replacement with oral testosterone undecanoate in hypogonadal men: a double blind controlled study. Clin. Endocrinol. (Oxf.) 14(1), 49-61 (1981)

38. M.L. Kottler, Y.Y. Chou, O. Chabre, N. Richard, C. Polge, S. Brailly-Tabard, P. Chanson, A. Guiochon-Mantel, I. Huhtaniemi, J. Young, A new FSHb mutation in a 29-year-old woman with primary amenorrhea and isolated FSH deficiency: functional characterization and ovarian response to human recombinant FSH. Eur. J. Endocrinol. 162(3), 633-641 (2010)

39. J. Tommiska, J. Toppari, K. Vaaralahti, J. K" ans" akoski, E.M. Laitinen, P. Noisa, A. Kinnala, H. Niinikoski, T. Raivio, PROKR2 mutations in autosomal recessive Kallmann syndrome. FertilSteril 99(3), 815-818 (2013)

40. G. Binder, R. Schweizer, P. Haber et al. Accuracy of endocrine tests for detecting hypogonadotropic hypogonadism in girls. J. Pediatrics 167(3), 674-678.e1 (2015)

41. A.A. Juul, A.M. Andersson, J.H. Petersen, T.K. Jensen, J. Muller et al. Serum inhibin A and inhibin B in healthy prepubertal, pubertal, and adolescent girls and adult women: relation to age, stage of puberty, menstrual cycle, follicle-stimulating hormone, luteinizing hormone, and estradiol levels. J. Clin. Endocrinol. Metab. 85, 1634-1640 (2000)
42. Y. Jacques, Cheng et al. Clinical management of congenital hypogonadotropic hypogonadism. Endocrine Rev. 40(2), 669-710 (2019)

43. T. Varimo, M. Hero, E.M. Laitinen, P.J. Miettinen, J. Tommiska, J. Kans " akoski, A. Juul, T. Raivio, Childhood growth in boys with congenital hypogonadotropic hypogonadism. Pediatr. Res. 79(5), 705-709 (2016)

44. J. Waldstreicher, S.B. Seminara, J.L. Jameson, A. Geyer, L.B. Nachtigall, P.A. Boepple, L.B. Holmes, W.F. Crowley Jr, The genetic and clinical heterogeneity of gonadotropin-releasing hormone deficiency in the human. J. Clin. Endocrinol. Metab. 81(12), 4388-4395 (1996)

45. D. Cassatella, S.R. Howard, J.S. Acierno et al. Congenital hypogonadotropic hypogonadism and constitutional delay of growth and puberty have distinct genetic architectures. Eur. J. Endocrinol. 178(4), 377-388 (2018)

46. J. Harrington, M.R. Palmert, Distinguishing constitutional delay of growth and puberty from isolated hypogonadotropic hypogonadism: critical appraisal of available diagnostic tests. J. Clin. Endocrinol. Metab. 97(9), 3056-3067 (2012)

47. T.Y. Segal, A. Mehta, A. Anazodo et al. Role of gonadotropinreleasing hormone and human chorionic gonadotropin stimulation tests in differentiating patients with hypogonadotropic hypogonadism from those with constitutional delay of growth and puberty. J. Clin. Endocrinol. Metab. 94(3), 780 (2009)

48. A. MartínezRamírez, M.G. Ropelato et al. Basal folliclestimulating hormone and peak gonadotropin levels after gonadotropin-releasing hormone infusion show high diagnostic accuracy in boys with suspicion of hypogonadotropic hypogonadism. J. Clin. Endocrinol. Metab. 95(6), 2811-2818 (2010)

49. J. Rohayem, E. Nieschlag, S. Kliesch et al. Inhibin B, AMH, but not INSL3, IGF1 or DHEAS support differentiation between constitutional delay of growth and puberty and hypogonadotropic hypogonadism. Andrology 3(5), 882-887 (2015)

50. Y. Jacques, X. Cheng, G.E. Papadakis et al. Clinical management of congenital hypogonadotropic hypogonadism. Endocr. Rev. 2, 2 (2018) 